\title{
Direct and Inverse Solutions with Geodetic Latitude in Terms of Longitude for Rhumb Line Sailing
}

\author{
Wei-Kuo Tseng, Michael A. Earle and Jiunn-Liang Guo \\ (Department of Merchant Marine, National Taiwan Ocean University) \\ (E-mail: wilco0917@yahoo.com.tw)
}

In this paper, equations are established to solve problems of Rhumb Line Sailing (RLS) on an oblate spheroid. Solutions are provided for both the inverse problem and the direct problem, thereby providing a complete solution to RLS. Development of these solutions was achieved in part by means of computer based symbolic algebra. The inverse solution described attains a high degree of accuracy for distance and azimuth. The direct solution has been obtained from a solution for latitude in terms of distance derived with the introduction of an inverse series expansion of meridian arc-length via the rectifying latitude. Also, a series to determine latitude at any longitude has been derived via the conformal latitude. This was achieved through application of Hermite's Interpolation Scheme or the Lagrange Inversion Theorem. Numerical examples show that the algorithms are very accurate and that the differences between original data and recovered data after applying the inverse or direct solution of RLS to recover the data calculated by the direct or inverse solution are very small. It reveals that the algorithms provided here are suitable for programming implementation and can be applied in the areas of maritime routing and cartographical computation in Graphical Information System (GIS) and Electronic Chart Display and Information System (ECDIS) environments.

\section{KEY WORDS \\ 1. Rhumb Line (RL). 2. Rectifying Latitude. 3. Conformal Latitude.}

First published online: 30 March 2012.

1. INTRODUCTION. In marine and air navigation, ships and aircraft sailing or flying on fixed compass headings may travel along Rhumb Lines (RL), hence knowledge of RL calculation is important. Mercator's projection (a normal aspect cylindrical conformal projection) has the unique property that RLs on the Earth's surface are projected as straight lines on the map.

Methods of calculating the course and the distance between two points from knowledge of their latitudes and longitudes, or calculating the latitude and the longitude of the arrival point from the course and the distance from a known departure point, are called 'sailings'. A RL appears as a straight line on a Mercator chart and as a spiral curve (Loxodrome) on a surface of an oblate spheroid. Both of these cut all the meridians at the same angle (Thomas, 1952; Williams, 1998). The distance for a RL on the navigation sphere is within $0.5 \%$ of the distance on the RL on the WGS84 spheroid (Earle, 2006) Figure 1. 


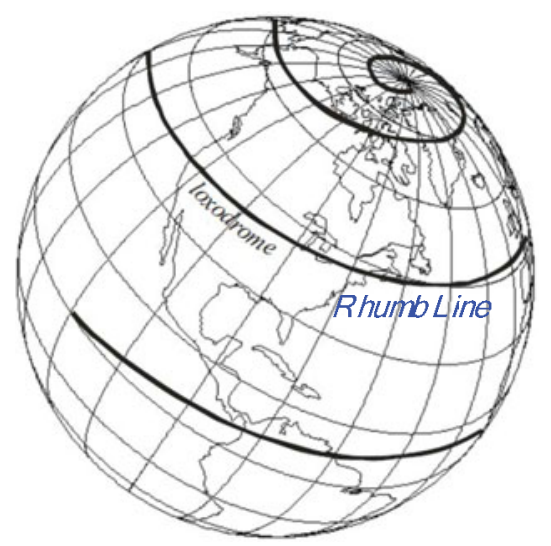

Figure 1. Rhumb Line (Loxodrome) on the Earth's surface.

In geodesy or navigation, the 'direct' problem (computing position given azimuth and distance from a known location) and the 'inverse' problem (computing azimuth and distance between known positions) are fundamental operations and can be likened to the equivalent operations of plane surveying. 'Radiations' (computing coordinates of points given bearings and distances radiating from a point of known coordinates) and 'joins'; (computing bearings and distances between points having known coordinates) are terms frequently used.

Solutions to the direct and inverse problem together constitute what is called the 'complete' solution. The terms 'direct' and 'inverse' in geodesy are usually associated with the geodesic, which is the unique curve defining the shortest path on the ellipsoid, but they can also be associated with other curves.

The direct solution for Rhumb Line Sailing (RLS) on the oblate spheroid is often treated by schemes that require iterative methods (Snyder, 1987; Tseng, 2006). Though adequate results have been achieved, the need for iterative methods has persisted without necessarily realizing higher accuracies (Bennett, 1996). Furthermore, interpolation for latitude in terms of longitude between end points of a RL on the spheroid has not yet been found in the literature. As a consequence of these observations, the complete solution to RLS presented here will include a method to determine latitude for any longitude along the RL. The accuracies attained can satisfy the requirement of ECDIS and GIS environments.

2. THE DIFFERENTIAL EQUATION OF THE RHUMB LINE. In rectangular coordinates, points such as $P$ on the oblate spheroid (Figure 2) have coordinates:

$$
\vec{P}=(x, y, z)=(a \cos \beta \cos \lambda, a \cos \beta \sin \lambda, b \sin \beta)
$$

where:

$\beta$ is reduced or parametric latitude.

$a$ is the semi-major axis.

$b$ is the semi-minor axis. 


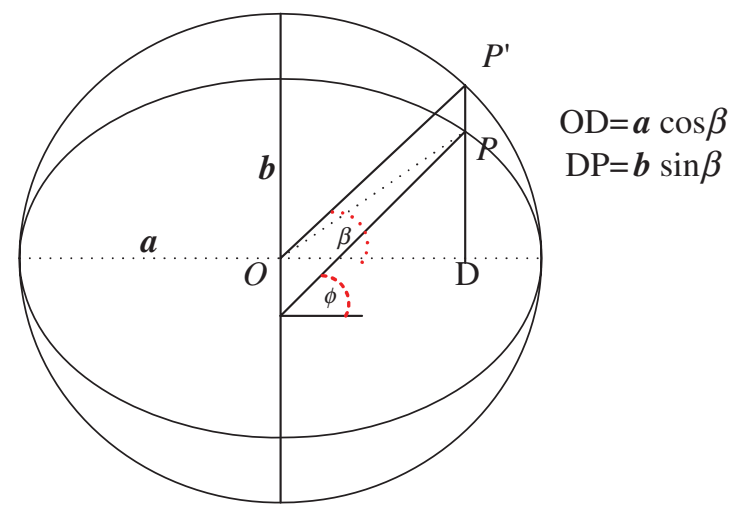

Figure 2. Meridian on a spheroid.

There are various ways of specifying the dimensions of the spheroid other than by its major and minor radii. The flattening, $f$, is defined by $f=1-b / a$, and the eccentricity $e$ by $e^{2}=1-b^{2} / a^{2}$. For the World Geodetic System 1984 (WGS 84), $a=6378137 \mathrm{~m}$ and $f=298 \cdot 257223563$.

A displacement of $d \beta$ in reduced latitude along the meridian is illustrated in Figure 3 . The displacements of three axes can be obtained by partial differentiating $P$ with respect to reduced latitude:

$$
\frac{d \vec{p}}{d \beta}=(-a \sin \beta \cos \lambda,-a \sin \beta \sin \lambda, b \cos \beta)
$$

The displacement parallel to the $\mathrm{z}$-axis is $b \cos \beta$ and the displacement towards the $\mathrm{z}$-axis is $-a \sin \beta$. The geodetic and reduced latitude are related by:

$$
\tan \varphi=\left(1-e^{2}\right)^{1 / 2} \tan \beta=(a / b) \tan \beta
$$

The radius of altitude parallel (Figure 3) is given by:

$$
O D=a \cos \beta=a \cos \varphi /\left(1-e^{2} \sin ^{2} \varphi\right)^{1 / 2}
$$

The result of a displacement of $d \beta$ in reduced latitude and of $d \lambda$ in longitude is illustrated in Figure 3.These displacements together cause a displacement $a \cos \beta d \lambda$ eastward and a displacement $a\left(1-e^{2} \cos ^{2} \beta\right)^{1 / 2} d \beta$ northward, respectively.

By Pythagoras's theorem the displacement $d s$ is given by:

$$
d s=\sqrt{\left(a^{2} \sin ^{2} \beta+b^{2} \cos ^{2} \beta\right) d \beta^{2}+a^{2} \cos ^{2} \beta d \lambda^{2}}
$$

Substituting Equation (3) into Equation (5) yields the following:

$$
d s=a \sqrt{\frac{\cos ^{2} \varphi \cdot d \lambda^{2}}{1-e^{2} \sin ^{2} \varphi}+\frac{\left(1-e^{2}\right)^{2} d \varphi^{2}}{\left(1-e^{2} \sin ^{2} \varphi\right)^{3}}}
$$

RLs are paths of constant true course. They thus satisfy the following:

$$
\tan \alpha=\frac{\cos \beta}{\sqrt{1-\varepsilon^{2} \cos ^{2} \beta}} \frac{d \lambda}{d \beta}=\frac{\cos \varphi\left(1-e^{2} \sin ^{2} \varphi\right)}{1-e^{2}} \frac{d \lambda}{d \varphi}
$$




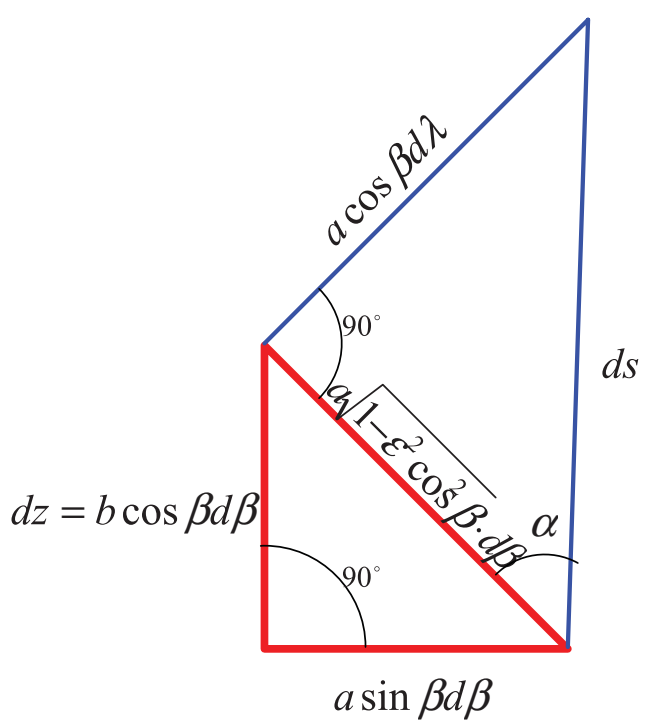

Figure 3. Triangles resulting from infinitesimal latitude and longitude changes.

This is most easily treated in geodetic latitude. Integrating Equation (7) yields:

$$
\lambda=\tan \alpha \cdot \operatorname{MP}\left(\varphi^{\prime}\right)
$$

where $\operatorname{MP}\left(\varphi^{\prime}\right)$, as used here, is the distance in meridional parts.

'Meridional parts' are defined as the length of the arc of a meridian between the Equator and a given parallel (geodetic latitude $\varphi^{\prime}$ ) on a Mercator chart, expressed in units of 1 minute of longitude at the Equator. Thus:

$$
M P\left(\varphi^{\prime}\right)=\ln \left[\tan \left(\left|\varphi^{\prime}\right| / 2+\pi / 4\right)\left(\frac{1-e \sin \left|\varphi^{\prime}\right|}{1-e \sin \left|\varphi^{\prime}\right|}\right)^{e / 2}\right]
$$

Combining Equation (6) and (7) with constant true course gives an integral for arc length along the RL.

$$
S(\varphi)=\sec \alpha \cdot a\left(1-e^{2}\right) \int_{0}^{\varphi}\left(1-e^{2} \sin ^{2} \theta\right)^{-3 / 2} d \theta
$$

where $R_{m}=a\left(1-\varepsilon^{2}\right)\left(1-\varepsilon^{2} \sin ^{2} \theta\right)^{-3 / 2}$ is the radius of curvature of meridian.

3. SOLUTIONS FOR RHUMB LINE SAILING. The arc-length of a meridian is the primary element for direct and inverse solutions to RLS. The above integral, Equation (6), can be approximated by a truncated series in the square of the eccentricity upon expanding the integrand in a binomial series:

$$
S(\varphi)=\sec \alpha \cdot a\left(1-e^{2}\right) \int_{0}^{\varphi}\left[\sum_{i=0}^{\infty}(-1)^{i}\left(\begin{array}{c}
-\frac{3}{2} \\
i
\end{array}\right)\left(e^{2} \sin ^{2} \theta\right)^{i}\right] d \theta
$$


In Equation (11) and Equation (12) below, each term of the binomial series for half integer multiples is defined by:

$$
\left(-\frac{3}{2}\right)=\frac{\frac{-3}{2} \cdot \frac{-5}{2} \ldots\left(\frac{-3}{2}-i+1\right)}{i \cdot(i-1) \ldots 1}
$$

Using trigonometric identities, the powers of the sine term may be reduced to combinations of cosine terms of the form $\cos (2 \cdot i \cdot \varphi)$. Collecting terms with the same cosine arguments and integrating gives the following series:

$$
S(\varphi)=\sec \alpha \cdot a\left(1-e^{2}\right)\left[M_{0} \varphi+\sum_{i=1}^{n} M_{2 i} \sin (2 \cdot i \cdot \varphi)\right]
$$

where:

$$
M_{0}=\sum_{k=0}^{\infty} \frac{(-1)^{k}}{2^{2 k}}\left(\begin{array}{c}
-\frac{3}{2} \\
k
\end{array}\right)\left(\begin{array}{c}
2 k \\
k
\end{array}\right) \varepsilon^{2 k} \text { and } M_{2 i}=\sum_{k=i}^{\infty} \frac{(-1)^{i+k}}{2^{2 k} i}\left(\begin{array}{c}
-\frac{3}{2} \\
k
\end{array}\right)\left(\begin{array}{c}
2 k \\
k-i
\end{array}\right) \varepsilon^{2 k}
$$

Expansion of coefficients for Equation (12) was facilitated using computer symbolic processing. These coefficients are shown below truncated at order $\mathrm{e}^{10}$ and $M_{10}$. Those up to $M_{8}$, first given by (Delambre, 1799) were used for confirmation of the results:

$$
M=\left[\begin{array}{c}
M_{0} \\
M_{2} \\
M_{4} \\
M_{6} \\
M_{8} \\
M_{10}
\end{array}\right]=\left[\begin{array}{cccccc}
1 & \frac{3}{4} & \frac{45}{64} & \frac{175}{256} & \frac{11025}{16384} & \frac{43659}{65536} \\
0 & -\frac{3}{8} & -\frac{15}{32} & -\frac{525}{1024} & -\frac{2205}{4096} & -\frac{72765}{131072} \\
0 & 0 & \frac{15}{256} & \frac{105}{1024} & \frac{2205}{16384} & \frac{10395}{65536} \\
0 & 0 & 0 & -\frac{35}{3072} & -\frac{105}{4096} & -\frac{10395}{262144} \\
0 & 0 & 0 & 0 & \frac{315}{131072} & \frac{3465}{524288} \\
0 & 0 & 0 & 0 & 0 & -\frac{693}{1310720}
\end{array}\right]\left[\begin{array}{c}
1 \\
e^{2} \\
e^{4} \\
e^{6} \\
e^{8} \\
e^{10}
\end{array}\right]
$$

3.1. RLS Direct Solution. The direct problem computes the arrival position given azimuth and distance to be travelled from a known location. The distance $\mathrm{S}\left(\varphi_{1}\right)$ to departure point $P_{1}$ from the Equator is first established and added to $\Delta S$, the distance to be travelled. Thus the overall distance from the Equator is:

$$
S(\varphi)=\Delta S+S\left(\varphi_{1}\right)
$$

Application of the Lagrange Inversion Theorem (Adams, 1921) or Hermite Interpolation Scheme produces the inverse solution of meridian arc-length so as to determine latitude from meridional distance. It is very difficult or even impossible, to derive the necessary high order derivatives by hand. Fortunately, modern computer aided symbolic algebra found in mathematical software packages mitigates the effort required (e.g., Mathematica ${ }^{\mathrm{TM}}$, Maple $^{\mathrm{TM}}$ or MATLAB $^{\mathrm{TM}}$ ).

Figure 4 shows the construction of the problem to be solved. Two known components of the RL having an azimuth $\alpha$ are shown in blue and red. The objective is to determine geodetic co-ordinates $\varphi, \lambda$ of the end point. 


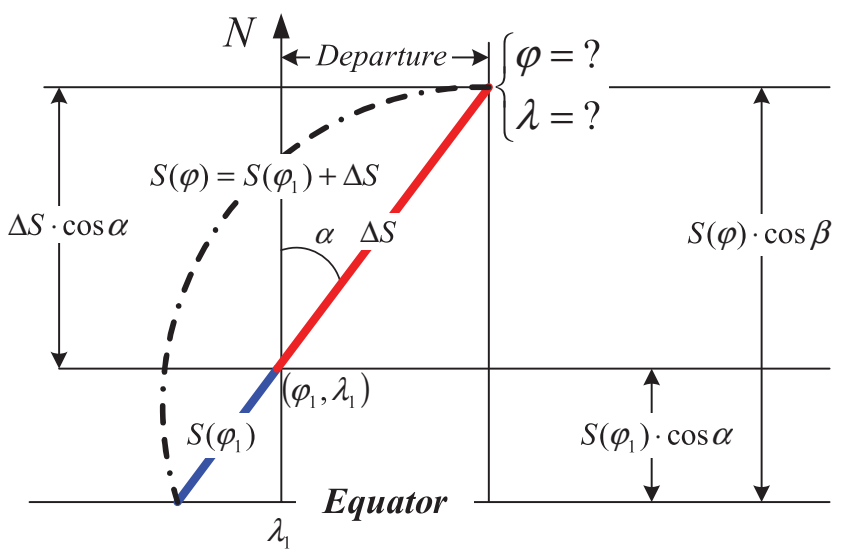

Figure 4. Direct problem of RLS.

Geodetic latitude as a function of distance from the Equator has been determined as just described through manipulation of Equation (10) to determine its inverse relationship. This inverse function gives geodetic latitude as in terms of the rectifying latitude $\mu$ :

$$
\varphi=\mu+\sum_{i=1}^{n} C_{2 i} \sin (2 \cdot i \cdot \mu)
$$

where distance $S(\varphi)$ is transformed into the rectifying latitude by:

$$
\mu=\frac{S(\varphi) \cdot \cos \alpha}{a\left(1-e^{2}\right) \cdot M_{0}}
$$

and for which the coefficients of $\sin (2 \cdot i \cdot \varphi)$ are:

$$
C=\left[\begin{array}{l}
C_{2} \\
C_{4} \\
C_{6} \\
C_{8}
\end{array}\right]=\left[\begin{array}{cccc}
3 / 8 & 3 / 16 & 213 / 2048 & 255 / 4096 \\
0 & 21 / 256 & 21 / 256 & 533 / 8192 \\
0 & 0 & 151 / 6144 & 151 / 4096 \\
0 & 0 & 0 & 1097 / 131072
\end{array}\right] \cdot\left[\begin{array}{c}
e^{2} \\
e^{4} \\
e^{6} \\
e^{8}
\end{array}\right]
$$

Once the geodetic end point or arrival latitude has been determined, the arrival longitude can be established from:

$$
\lambda=\lambda_{1}+\tan \alpha \cdot\left[M P(\varphi)-M P\left(\varphi_{1}\right)\right]
$$

The tangent of true course $(\tan \alpha)$ becomes infinity when true course is East or West. In which case, RL distance is along a parallel at the latitude of departure. Latitude and longitude of end point are then set by:

$$
\left\{\begin{array}{l}
\varphi=\varphi_{1} \\
\lambda=\lambda_{1}+\frac{\Delta S\left(1-e^{2} \sin ^{2} \varphi_{1}\right)^{1 / 2}}{a \cos \varphi_{1}}
\end{array}\right.
$$

3.2. RLS Inverse Solution. The inverse problem, as indicated in Figure 5, determines the true azimuth and the distance between two points given the departure 


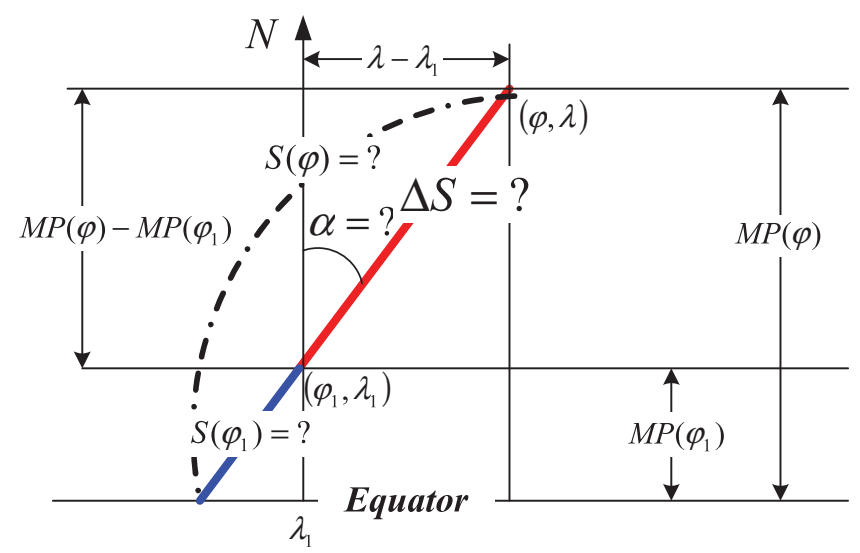

Figure 5. Inverse problem of RLS.

and destination coordinates. Since the end points are known, azimuth is determined from application of Equation (8) to the end point latitudes to obtain the true course of the RL.

$$
\alpha=\operatorname{atan} 2\left[\Delta \lambda, \quad M P(\varphi)-M P\left(\varphi_{1}\right)\right]
$$

Equation (21) maps an angle $\lambda-\lambda_{1}$ (in radians) to the interval $\left[\begin{array}{ll}-\pi & \pi\end{array}\right]$ and gives $\Delta \lambda$ correctly as:

$$
\Delta \lambda=\pi \cdot\left\{\frac{\left|\lambda-\lambda_{1}\right|}{\pi}-2 \cdot \operatorname{ceil}\left[\left(\frac{\left|\lambda-\lambda_{1}\right|}{\pi}-1\right) / 2\right]\right\} \cdot \operatorname{sign}\left(\lambda-\lambda_{1}\right)
$$

where ceil $(x)$ rounds the elements of $x$ to the smallest integer greater than or equal to $x$.

The RL distance between two latitudes is next calculated with Equation (12). So then:

$$
\Delta S=S(\varphi)-S\left(\varphi_{1}\right)
$$

Since the tangent of true course $(\tan \alpha)$ becomes infinity for East or West courses, Equation (12) cannot be applied to calculate the distance. In which case, the RL distance is the arc-length of part of the parallel at the latitude of departure given by:

$$
S(\lambda)=a\left(\lambda-\lambda_{1}\right) \frac{\cos \varphi_{1}}{\left(1-e^{2} \sin \varphi_{1}\right)^{1 / 2}}
$$

3.3. Interpolation of the Rhumb Line at Other Longitudes. Once a RL has been determined between two end points, it may be required to determine the latitude at one or more other longitudes between end points. Equation (8) provides the longitude in terms of latitude. The geodetic latitude of any point along the RL can be determined once the longitude and true course have been specified. Figure 6 shows a $\mathrm{RL}$ for which latitude $\varphi$ is to be found for an arbitrary longitude $\lambda$. 


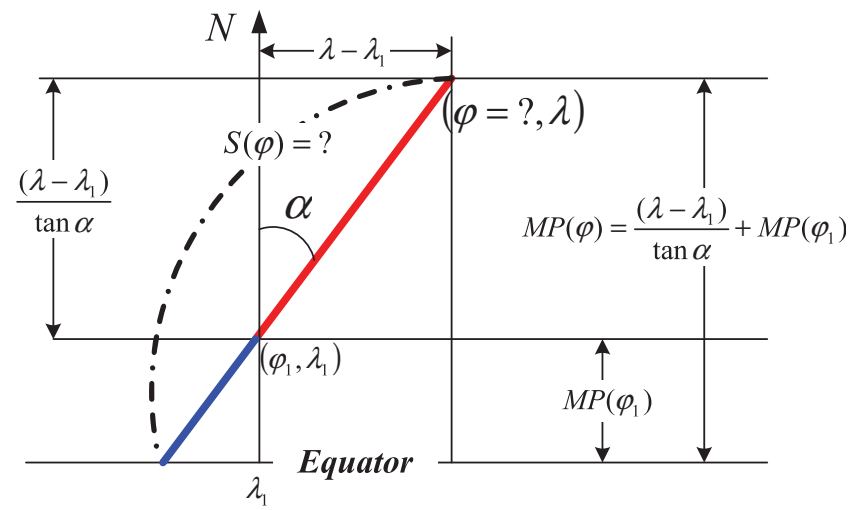

Figure 6. Latitude in terms of longitude.

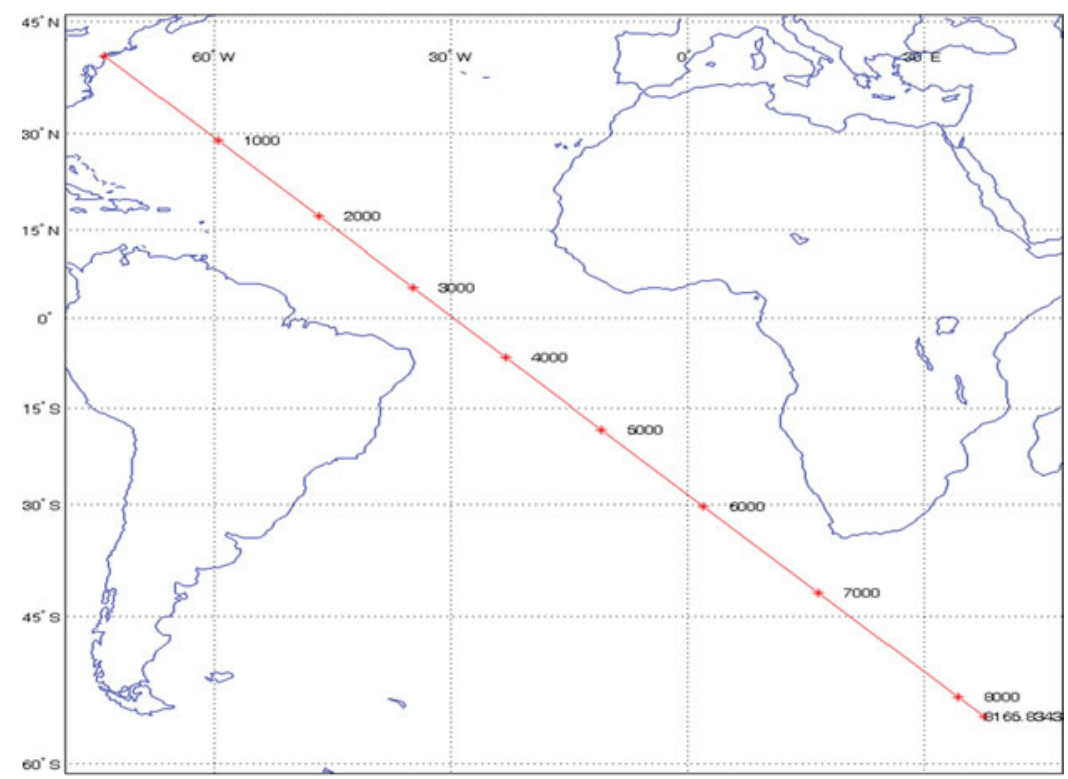

Figure 7. A ship steers from $F\left(40^{\circ} 43^{\prime} N, 74^{\circ} 00^{\prime} W\right)$ to $T\left(55^{\circ} 45^{\prime} S, 37^{\circ} 37^{\prime} E\right)$.

First, application of Equation (8) gives the meridional parts at a given longitude along RL:

$$
M P(\varphi)=\frac{\left(\lambda-\lambda_{1}\right)}{\tan \alpha}+M P\left(\varphi_{1}\right)
$$

Secondly, the conformal latitude $\delta$ associated with $\operatorname{MP}\left(\varphi_{1}\right)$ can be obtained from the following:

$$
\ln [\tan (\delta / 2+\pi / 4)]=M P(\varphi)
$$


Table 1. Positions differing in distance and recovered distances and courses. Applied Equations: (15), (18), (20) and (22).

True course: 134.9794964. Total Distance: $8165 \cdot 8343419 \mathrm{~nm}$. *: Recovered Data -/W, +/E

\begin{tabular}{lcrrrrrc}
\hline Distance & Course & \multicolumn{1}{c}{ Latitude } & Longitude & \multicolumn{1}{c}{ Distance* } & \multicolumn{1}{c}{ Error } & Course* & Error \\
\hline 1000 & $134 \cdot 9795$ & $28 \cdot 91651$ & $-59 \cdot 63111$ & $999 \cdot 9999999983$ & $1 \cdot 742 \mathrm{E}-08$ & $134 \cdot 9795$ & $0 \cdot 000 \mathrm{E}+00$ \\
2000 & $134 \cdot 9795$ & $17 \cdot 09592$ & $-46 \cdot 82160$ & $1999 \cdot 999999995$ & $5 \cdot 150 \mathrm{E}-09$ & $134 \cdot 9795$ & $0 \cdot 000 \mathrm{E}+00$ \\
3000 & $134 \cdot 9795$ & $5 \cdot 26174$ & $-34 \cdot 80436$ & $3000 \cdot 000000000$ & $1 \cdot 000 \mathrm{E}-11$ & $134 \cdot 9795$ & $0 \cdot 000 \mathrm{E}+00$ \\
4000 & $134 \cdot 9795$ & $-6 \cdot 57686$ & $-23 \cdot 01453$ & $4000 \cdot 000000001$ & $-5 \cdot 498 \mathrm{E}-10$ & $134 \cdot 9795$ & $0 \cdot 000 \mathrm{E}+00$ \\
5000 & $134 \cdot 9795$ & $-18 \cdot 40995$ & $-10 \cdot 93931$ & $5000 \cdot 000000007$ & $-6 \cdot 620 \mathrm{E}-09$ & $134 \cdot 9795$ & $0 \cdot 000 \mathrm{E}+00$ \\
6000 & $134 \cdot 9795$ & $-30 \cdot 22855$ & $1 \cdot 99987$ & $6000 \cdot 000000019$ & $-1 \cdot 929 \mathrm{E}-08$ & $134 \cdot 9795$ & $0 \cdot 000 \mathrm{E}+00$ \\
7000 & $134 \cdot 9795$ & $-42 \cdot 02616$ & $16 \cdot 60643$ & $7000 \cdot 000000031$ & $-3 \cdot 148 \mathrm{E}-08$ & $134 \cdot 9795$ & $0 \cdot 000 \mathrm{E}+00$ \\
8000 & $134 \cdot 9795$ & $-53 \cdot 79982$ & $34 \cdot 23991$ & $8000 \cdot 000000041$ & $-4 \cdot 085 \mathrm{E}-08$ & $134 \cdot 9795$ & $0 \cdot 000 \mathrm{E}+00$ \\
$8165 \cdot 83$ & $134 \cdot 9795$ & $-55 \cdot 75000$ & $37 \cdot 61667$ & $8165 \cdot 834341902$ & $-4 \cdot 229 \mathrm{E}-08$ & $134 \cdot 9795$ & $0 \cdot 000 \mathrm{E}+00$
\end{tabular}

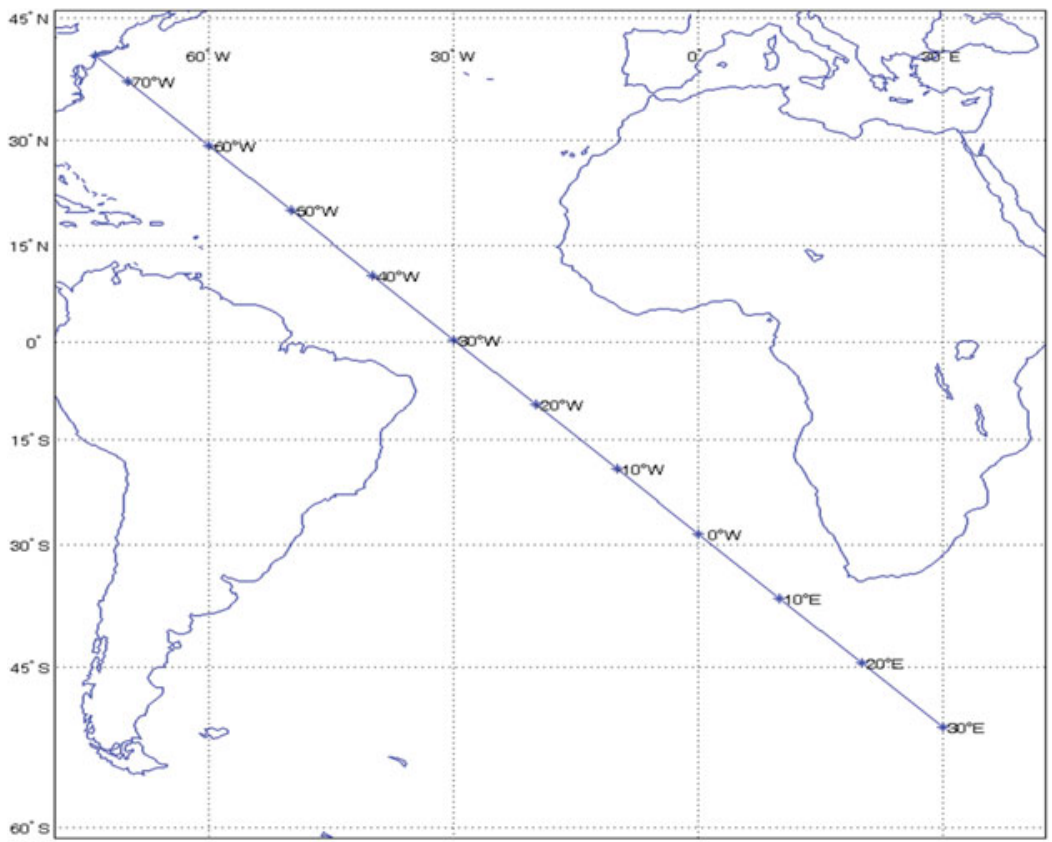

Figure 8. Sampling by Given Longitude.

which is equivalent to:

$$
\delta=2 \operatorname{atan}(\exp (M P(\varphi)))-\pi / 2
$$

or:

$$
\delta=\operatorname{asin}(\tanh (M P(\varphi))
$$

The inversion of Equation (27) has once again been accomplished via the Lagrange Inversion Theorem (Adams, 1927) or the Hermite Interpolation Scheme. Computer symbolic algebra was again used to determine coefficients of a series for conformal 
Table 2. Latitude in terms of Longitude and Recovered Longitude. Applied equations: (18), (24), (27), and (28).

\begin{tabular}{lrrr}
\hline Longitude & Latitude & Recovered Longitude & \multicolumn{1}{c}{ Error } \\
\hline-70 & $37 \cdot 60573351$ & $-70 \cdot 0000000000372$ & $3 \cdot 720 \mathrm{E}-11$ \\
-60 & $29 \cdot 24033053$ & $-59 \cdot 9999999999941$ & $-5 \cdot 898 \mathrm{E}-12$ \\
-50 & $20 \cdot 12376295$ & $-49 \cdot 9999999999124$ & $-8 \cdot 760 \mathrm{E}-11$ \\
-40 & $10 \cdot 43718086$ & $-39 \cdot 9999999998931$ & $-1 \cdot 069 \mathrm{E}-10$ \\
-30 & $0 \cdot 43596702$ & $-29 \cdot 9999999999942$ & $-5 \cdot 802 \mathrm{E}-12$ \\
-20 & $-9 \cdot 57868828$ & $-20 \cdot 0000000001025$ & $1 \cdot 025 \mathrm{E}-10$ \\
-10 & $-19 \cdot 30355896$ & $-10 \cdot 0000000000935$ & $9 \cdot 350 \mathrm{E}-11$ \\
0 & $-28 \cdot 47787520$ & $-0 \cdot 0000000000124$ & $1 \cdot 238 \mathrm{E}-11$ \\
10 & $-36 \cdot 91346593$ & $10 \cdot 0000000000359$ & $-3 \cdot 590 \mathrm{E}-11$ \\
20 & $-44 \cdot 50384451$ & $20 \cdot 0000000000292$ & $-2 \cdot 920 \mathrm{E}-11$ \\
30 & $-51 \cdot 21555600$ & $30 \cdot 0000000000034$ & $-3 \cdot 400 \mathrm{E}-12$ \\
\hline
\end{tabular}

latitude. The resulting expression for geodetic latitude in terms of conformal latitude (Adams, 1927; Thomas, 1952; Snyder, 1987) is then:

$$
\varphi=\delta+\sum_{i=1}^{n} K_{2 i} \sin (2 \cdot i \cdot \delta)
$$

Expanding to $O\left(\mathrm{e}^{8}\right)$, terms for $K$ are approximately given by.

$$
K=\left[\begin{array}{l}
K_{2} \\
K_{4} \\
K_{6} \\
K_{8}
\end{array}\right]=\left[\begin{array}{cccc}
\frac{1}{2} & \frac{5}{24} & \frac{1}{12} & \frac{13}{360} \\
0 & \frac{7}{48} & \frac{29}{240} & \frac{811}{11520} \\
0 & 0 & \frac{7}{120} & \frac{81}{1120} \\
0 & 0 & 0 & \frac{4279}{161280}
\end{array}\right]\left[\begin{array}{c}
e^{2} \\
e^{4} \\
e^{6} \\
e^{8}
\end{array}\right]
$$

4. NUMERICAL TEST. A ship steers from a departure point at $F\left(40^{\circ} 43^{\prime} N\right.$, $\left.74^{\circ} 00^{\prime} \mathrm{W}\right)$ to a destination at $T\left(55^{\circ} 45^{\prime} \mathrm{S}, 37^{\circ} 37^{\prime} \mathrm{E}\right)$ along the RL on the WGS84 Earth (Figure 7). Course and distance are initially determined from the given end points using Equations (20) and (22). Latitudes and longitudes of waypoints beyond departure point at $F$ at distances of $1000,2000, \ldots$ nautical miles are then found after using Equations (15) and (18). Results are shown in Table 1.

For each value of latitude and longitude calculated at each waypoint, Equations (20) and (22) are applied to recover their corresponding values of course and distance as shown in Table 1 in the columns for Course* and Distance*. It can be seen that the resulting differences are negligible as shown in Table 1, Column 6 (Distance Error) and Column 8 (Course Error).

In another test for recovered longitude, the latitudes of waypoints beyond the departure point $F$ located at $70^{\circ} \mathrm{W}, 60^{\circ} \mathrm{W}, 50^{\circ} \mathrm{W}, \ldots, 30^{\circ} \mathrm{E}$ (Figure 8) are first found after using Equations (24), (27), and (28) and are shown in Table 2, column 2 (Latitude).

On applying Equation (18) using the calculated latitude shown in Table 2, column 2 (Latitude), the recovered longitude was then calculated as shown in Table 2, column 
3. The resulting differences when compared with the given values of Table 2, column 1 are also negligible as shown in Table 2, column 4 (Error).

5. CONCLUSIONS. Accurate solutions to the direct and inverse problems of Rhumb Line Sailing (RLS) have been described and demonstrated. These two solutions provide useful alternatives to previous approaches in that they require no recourse to integrals or iteration methods. The solution for latitude in terms of longitude has also been described and demonstrated. This was achieved by application of an inverse expansion of conformal latitude that gives very accurate latitude in terms of longitude without iteration.

These three series along with the formula for meridional parts are seen to be simple, direct and computationally efficient. They provide positioning accuracies involving distance, position, and true course that are in the sub-metre range and which are also commensurate with the current levels of accuracy achieved by Global Navigation Satellite Systems (GNSS), while at the same time, they provide a complete solution to RLS. The algorithms provided here are easily incorporated into computer software and are well suited to vessel route planning and cartographical computation in Graphical Information System (GIS) and Electronic Chart Display and Information System (ECDIS) environments.

\section{ACKNOWLEDGEMENTS}

This work was supported in part by the National Science Council of Taiwan, Republic of China, under grant NSC 98-2410-H-019-019-, NSC 99-2410-H-019-023-, and NSC 100-2410H-019-018-.

\section{REFERENCES}

Adams, O.S. (1921). Latitude Developments Connected With Geodesy and Cartography. United States Coast and Geodetic Survey, Special Publication 67, Washington, USA.

Bennett, G. G. (1996). Practical RL Calculations on the Spheroid. Journal of Navigation, 49, 112-119.

Delambre, J. B. J. (1799). Méthodes Analytiques pour la Détermination d'un Arc du Méridien; précédées d'un mémoire sur le même sujet par A. M. Legendre. De L’Imprimerie de Crapelet, Paris.

Earle, M. A. (2006). Sphere to Spheroid Comparisons. The Journal of Navigation, 59, 491-496.

Snyder, J. P. (1987). Map Projections-A Working Manual. Professional Paper No. 1395. U.S. Geological Survey.

Thomas, P. D. (1952). Conformal Projections in Geodesy and Cartography. US Coast and Geodetic Survey, Publication No. 251.

Tseng, W. K (2006). Vector Solutions for the Sailing. National Taiwan Ocean University, Taiwan.

Williams, R. (1998). Geometry of Navigation. Horwood Publishing Ltd, Chichester, UK. 